Article

\title{
Functional and Kinetic Comparison of Alanine Cysteine Serine Transporters ASCT1 and ASCT2
}

\author{
Jiali Wang (D), Yang Dong and Christof Grewer* \\ Department of Chemistry, Binghamton University, 4400 Vestal Parkway East, Binghamton, NY 13902, USA; \\ jwang204@binghamton.edu (J.W.); ydong45@binghamton.edu (Y.D.) \\ * Correspondence: cgrewer@binghamton.edu
}

\begin{abstract}
Neutral amino acid transporters ASCT1 and ASCT2 are two SLC1 (solute carrier 1) family subtypes, which are specific for neutral amino acids. The other members of the SLC1 family are acidic amino acid transporters (EAATs $1-5$ ). While the functional similarities and differences between the EAATs have been well studied, less is known about how the subtypes ASCT1 and 2 differ in kinetics and function. Here, by performing comprehensive electrophysiological analysis, we identified similarities and differences between these subtypes, as well as novel functional properties, such as apparent substrate affinities of the inward-facing conformation (in the range of $70 \mu \mathrm{M}$ for L-serine as the substrate). Key findings were: ASCT1 has a higher apparent affinity for $\mathrm{Na}^{+}$, as well as a larger $\left[\mathrm{Na}^{+}\right]$dependence of substrate affinity compared to ASCT2. However, the general sequential $\mathrm{Na}^{+}$/substrate binding mechanism with at least one $\mathrm{Na}^{+}$binding first, followed by amino acid substrate, followed by at least one more $\mathrm{Na}^{+}$ion, appears to be conserved between the two subtypes. In addition, the first $\mathrm{Na}^{+}$binding step, presumably to the $\mathrm{Na} 3$ site, occurs with high apparent affinity $(<1 \mathrm{mM})$ in both transporters. In addition, ASCT1 and 2 show different substrate selectivities, where ASCT1 does not respond to extracellular glutamine. Finally, in both transporters, we measured rapid, capacitive charge movements upon application and removal of amino acid, due to rearrangement of the translocation equilibrium. This charge movement decays rapidly, with a time constant of 4-5 ms and recovers with a time constant in the $15 \mathrm{~ms}$ range after substrate removal. This places a lower limit on the turnover rate of amino acid exchange by these two transporters of $60-80 \mathrm{~s}^{-1}$.
\end{abstract}

Keywords: neutral amino acid transporter; electrophysiology; kinetics; rapid solution exchange

\section{Introduction}

ASCTs (alanine serine cysteine transporters) belong to the SLC1 family of membrane Received: 16 December 2021 Accepted: 7 January 2022 Published: 11 January 2022

Publisher's Note: MDPI stays neutral with regard to jurisdictional claims in published maps and institutional affiliations.

Copyright: (c) 2022 by the authors. Licensee MDPI, Basel, Switzerland. This article is an open access article distributed under the terms and conditions of the Creative Commons Attribution (CC BY) license (https:// creativecommons.org/licenses/by/ $4.0 /)$. transport proteins. Two subtypes of this family, ASCT1 and ASCT2 (SLC1A4 and SLC1A5) are neutral amino acid transporters, in contrast to the remaining five members of the family (excitatory amino acid transporters, EAATs), which transport acidic amino acids [1-7]. ASCT2 was previously shown to function as a $\mathrm{Na}^{+}$-dependent neutral amino acid exchanger, which requires the presence of $\mathrm{Na}^{+}$, but does not use the $\mathrm{Na}^{+}$gradient as a driving force for amino acid uptake [2-5,8]. In this mechanism, extracellular amino acid is exchanged with intracellular amino acid, without the ability of the empty transporter to complete the transport cycle.

In previous studies, a high expression level of ASCT2 was observed in cancer cells, suggesting an association with cancer metabolism. It was suggested to directly or indirectly provide energy and nitrogen resources to rapidly growing cells, by taking up glutamine [9-12]. In addition, ASCT1 and 2 may be involved in neuronal disorders. For example, SLC1A4 is the pathogenic gene of a neurologic disorder manifesting in intellectual disability, postnatal microcephaly, spasticity and thin corpus callosum [13].

Within the SLC1 family, the various subtypes share a high sequence similarity and identity [14]. ASCT1 and 2 share 57\% sequence identity and ASCTs share up to $29 \%$ sequence identity with EAATs $[12,14]$. The substrate binding site, however, shows distinct 
differences, accounting for the diverging substrate specificities of ASCTs and EAATs. The excitatory amino acid transporters have a positively-charged side chain (highly conserved arginine) in the binding site, which forms an ion pair with the negatively charged amino acid side chain [15-17]. In the ASCTs, this cationic side chain is replaced with a neutral amino acid, for example cysteine in ASCT2. In addition to diverging amino acid substrate specificities, EAATs and ASCTs also have significant differences in function. For example, EAATs do not function as exchangers and, in contrast to ASCTs, counter-transport $\mathrm{K}^{+}[18,19]$. Even among the EAAT subtypes, functional differences exist. For example, EAATs 4-5 show slower kinetics and distinct voltage dependence of transport compared to EAATs 1-3 [20-25]. In addition, cation selectivities are different within the EAATs, in particular with respect to the ability to transport $\mathrm{Li}^{+}[18,26,27]$. Therefore, it is possible that ASCT1 and 2 also show distinct functional properties, although little is known about the details of kinetics and voltage dependence of ASCT1 amino acid exchange.

While the structure of ASCT1 is not known, structures of ASCT2 have recently been published in both the substrate-bound inward- and outward-facing conformations, as well as in complex with a competitive inhibitor [28-31]. These structures highlight the importance of the hairpin loop 2 (HP2), which locks the substrate into the binding site and acts as both the internal and external gate in the elevator-like alternating access transport mechanism. However, the kinetic properties of the inward-facing amino acid binding site, for example the apparent $K_{\mathrm{m}}$ values for substrates, are not well characterized [32].

Another important topic is substrate selectivity. In a previous study, it was shown that, in contrast to ASCT2, ASCT1 does not recognize glutamine as a substrate [3]. However, apart from a review article published while this work was in preparation [14], no other reports have focused on the functional similarities and differences between ASCT1 and ASCT2. Here, we applied electrophysiological techniques to comprehensively study the kinetic properties of ASCT1 and 2. Our results demonstrate that ASCT1 has stronger apparent affinity for amino acid substrates alanine and serine than ASCT2. In addition, $\mathrm{Na}^{+}$interacts more strongly with ASCT1 than with ASCT2. Finally, the leak anion conductance is much less pronounced in ASCT1 than ASCT2. Interestingly, ASCT1 and ASCT2 showed similarity in kinetic properties when probing the electrogenic steps associated with substrate translocation. Both transporters exhibited amino acid-induced rapid charge movement, which recovered upon substrate removal with a time constant in the $15 \mathrm{~ms}$ range, placing an upper limit on the ASCT1 and ASCT2 cycle time.

\section{Materials and Methods}

\subsection{Cell Culture and Transfection}

HEK293 cells (American Type Culture Collection No. CRL 1573) were cultured as described previously. Cell cultures were transiently transfected with wild-type or mutant hASCT1, hASCT2 cDNAs, inserted into a modified pBK-CMV-expression plasmid. Transfection were performed according Jetprime transfection reagent and protocol (Polyplus). The cells were transfected after 24-36 h then used for electrophysiological analysis.

\subsection{Electrophysiology}

Currents associated with alanine serine cysteine transporters were measured in the whole-cell current recording configuration. Whole-cell currents were recorded with an EPC7 patch-clamp Amplifier (ALA Scientific, Westbury, NY, USA) under voltage-clamp conditions. The resistance of the recording electrode was 3-6 M $\Omega$. Series resistance was not compensated because of the small whole-cell currents carried by ASCTs. The composition of the solutions for measuring amino acid exchange currents in the anion conducting mode was: $140 \mathrm{mM}$ NaMes (Mes = methanesulfonate), $2 \mathrm{mM} \mathrm{MgGluconate} 2,2 \mathrm{mM}$ $\mathrm{CaMes}_{2}, 10 \mathrm{mM}$ 4-(2-Hydroxyethyl)piperazine-1-ethanesulfonic acid (HEPES), pH 7.3 (extracellular) and $130 \mathrm{mM} \mathrm{NaSCN}$ (SCN = thiocyanate), $10 \mathrm{mM}$ Ser, $2 \mathrm{mM} \mathrm{MgGluconate}{ }_{2}$, $5 \mathrm{mM}$ Ethylene glycol-bis(2-aminoethylether)- $N, N, N^{\prime}, N^{\prime}$-tetraacetic acid (EGTA), $10 \mathrm{mM}$ 
HEPES, pH 7.3 (intracellular), as published previously [19]. For the measurement of the transport component of the current, intracellular $\mathrm{SCN}^{-}$was replaced with the $\mathrm{Mes}^{-}$anion.

\subsection{Voltage-Jump Experiments}

Voltage jumps $(-100$ to $+60 \mathrm{mV})$ were applied to perturb the translocation equilibrium and to determine the voltage dependence of the anion conductance. To determine ASCT-specific currents, external solution contained $140 \mathrm{mM}$ NaMes in the presence of varying concentrations of amino acid substrate. The internal solution contained $130 \mathrm{mM}$ $\mathrm{NaSCN}$ in the presence of $10 \mathrm{mM}$ amino acid substrate. Competitive blocker (R)-gamma-(4biphenylmethyl)-L-proline [33] was used in control voltage jump experiments, yielding the unspecific current component, which was subtracted from the total current. Capacitive transient compensation and series resistance compensation of up to $80 \%$ was employed using the EPC-7 amplifier. Non-specific transient currents were subtracted in Clampfit software (Molecular Devices).

\subsection{Rapid Solution Exchange}

Fast solution exchanges were performed using the SF-77B (Warner Instruments, LLC, Holliston, MA, USA) piezo-based solution exchanger, allowing a time resolution in the 10-20 ms range. Amino acid substrate was applied through a theta capillary glass tubing (TG200-4, OD $=2.00 \mathrm{~mm}$, ID = $1.40 \mathrm{~mm}$. Warner Instruments, LLC, MA, USA), with the tip of the theta tubing pulled to a diameter of $350 \mu \mathrm{m}$ and positioned at $0.5 \mathrm{~mm}$ to the cell [34]. For paired-pulse experiments, currents were recorded with varying interval time after removal of amino acid, starting at $10 \mathrm{~ms}$.

\subsection{Data Analysis}

The data analysis was performed in Microsoft Excel and Microcal Origin software. Error bars are shown as mean \pm stand deviation, collected from recordings of 6 to 10 cells, for statistical analysis. To determine substrate and $\mathrm{Na}^{+}$apparent $K_{\mathrm{m}}$ values, non-linear curve fitting was used with a Michaelis-Menten-like equation, $\mathrm{I}=\mathrm{I}_{\max } \times\left[\right.$ substrate] $/\left(K_{\mathrm{m}}+\right.$ [substrate]), where $I_{\max }$ is the current at saturating substrate concentration.

Transient signals of piezo-based solution-exchange results were analyzed in Clampfit software (Axon Instruments) by fitting with a sum of two exponential components. $\mathrm{I}=\mathrm{I}_{1} \cdot \exp \left(-\mathrm{t} / \tau_{\text {rise }}\right)+\mathrm{I}_{2} \cdot \exp \left(-\mathrm{t} / \tau_{\text {decay }}\right)$. Here, $\mathrm{I}$ is the current amplitude, $\tau$ the time constant and $\mathrm{t}$ the time.

\section{Results}

\subsection{ASCT1 Binds Amino Acid Substrate with Higher Apparent Affinity than ASCT2 in the Outward-Facing State}

To test the function of ASCTs, including apparent substrate affinities, we selected the HEK293 cell expression system for transfection with ASCT cDNAs, together with electrophysiological analysis in the whole cell recording configuration. In this system, alanine did not elicit currents in non-transfected cells. Typical currents recorded from human ASCT2 transfected cells in response to extracellular solution exchange to various concentrations of serine are shown in Figure 1A. As expected, application of serine activated the ASCT2 anion conductance in the presence of the intracellular anion, $\mathrm{SCN}^{-}$, indicating the population of the substrate-induced anion-conducting state. No steady-state currents were observed in the absence of permeating anions and at zero-trans conditions for substrate and sodium, as discussed later (Section 3.5). Inward currents were concentration dependent and were analyzed with a Michaelis-Menten-like equation, to yield the apparent $K_{\mathrm{m}}$ for the substrate, serine (Figure 1B) as $350 \pm 60 \mu \mathrm{M} . K_{\mathrm{m}}$ values for other amino acid substrates in comparison with ASCT1 are summarized in Figure 1C, indicating that substrate apparent affinities are 2-5 times higher in ASCT1 than in ASCT2. Consistent with previous results, L-glutamine did not generate currents in ASCT1-expressing cells. These data confirm 
previous results that neutral amino acids serine and alanine are major substrates for ASCTs but glutamine only binds to ASCT2.
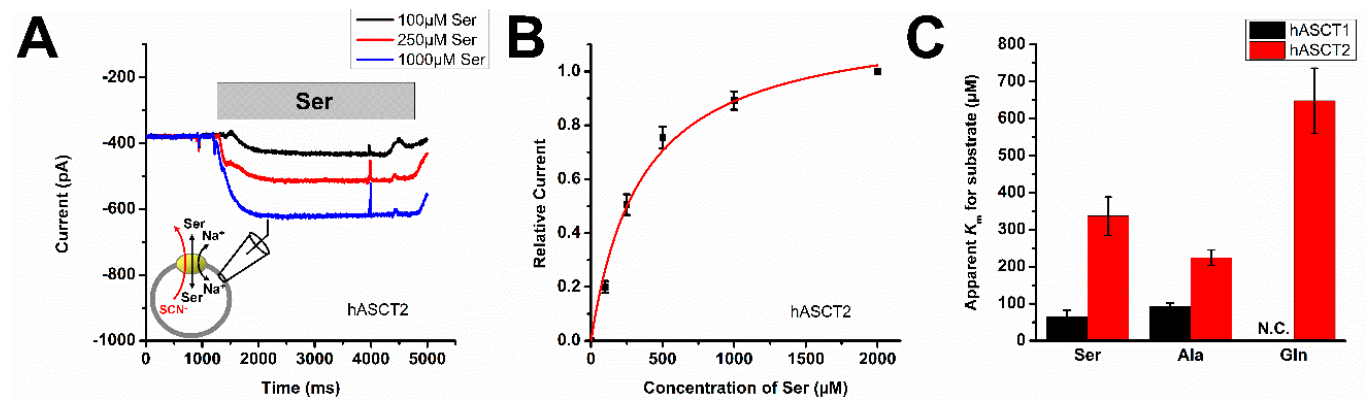

Figure 1. ASCT1 and 2 apparent substrate affinity and selectivity. (A) Typical current recording example with ASCT2 at varying serine concentrations (time of application indicated by the grey bar). (B) The serine apparent $K_{\mathrm{m}}$ was determined as $350 \pm 60 \mu \mathrm{M}$ for ASCT2 by fit of a Michaelis-Mentenlike equation (red line). Relative current was determined by normalizing all current responses to the response obtained at $2 \mathrm{mM}$ serine. (C) Comparison of $K_{\mathrm{m}}$ values for three substrates of ASCT1 and ASCT2. N.C. indicates no current was observed. Whole cell current recording experiments were performed under homo-exchange conditions: $130 \mathrm{mM} \mathrm{NaSCN}$ and $10 \mathrm{mM}$ of serine in the pipette solution, the extracellular solution was $140 \mathrm{mM}$ NaMes and variable concentration of substrate at $\mathrm{V}=0 \mathrm{mV}$.

\subsection{Leak Anion Conductance Is Less Pronounced in ASCT1 than in ASCT2}

ASCT anion conductance was tested using $\mathrm{SCN}^{-}$as a highly permeable anion at the intracellular side of the membrane. As expected, application of amino acid substrate at a concentration of $500 \mu \mathrm{M}$ generated inward current, which increased with negative membrane potential (Figure 2A, voltage jump protocol illustrated in the top panel). This inward current is caused by SCN- leaving the cell. Steady-state current was then plotted as a function of the membrane voltage, as shown in the I-V curves in Figure 2B,C. Serine induced the largest anion current in both transporters, while glutamine was ineffective in generating anion current in ASCT1 at any voltage tested. Interestingly, ASCT1 showed a much smaller leak anion current, relative to the substrate-induced current, than ASCT2. This current was observed in the absence of amino acid substrate, but in the presence of $\mathrm{Na}^{+}$. It is specific for ASCTs, as background was subtracted using a competitive inhibitor, which blocks the leak anion conductance.

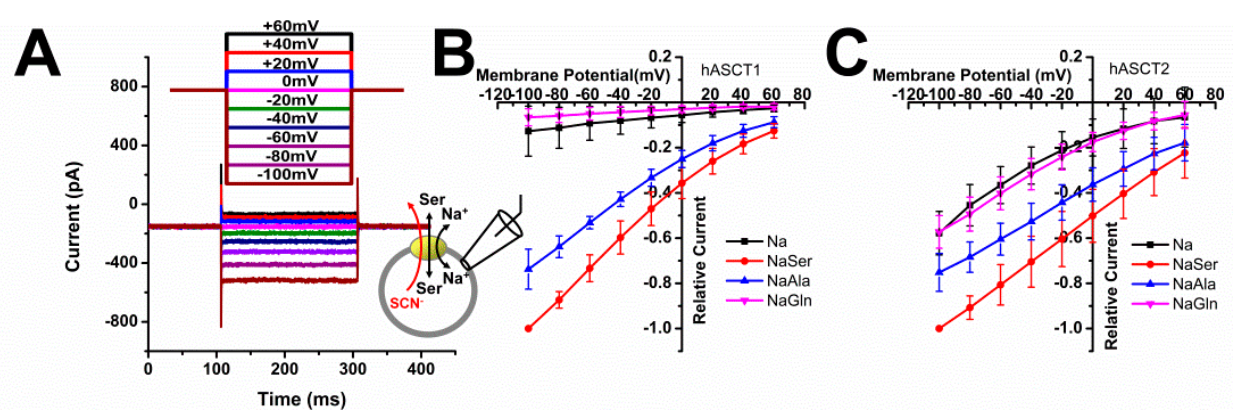

Figure 2. Voltage dependence of substrate-induced and leak anion currents. (A) Typical current recording in response to voltage jumps (from -100 to $+60 \mathrm{mV}$, in increments of $20 \mathrm{mV}$ for the duration of $200 \mathrm{~ms}$, voltage protocol shown at the top) from an ASCT1-transfected cell in the presence of extracellular NaMes and intracellular NaSCN with $10 \mathrm{mM}$ serine. $400 \mu \mathrm{M}$ (R)-gamma-(4Biphenylmethyl)-L-proline was used to eliminate the unspecific background currents by subtraction. The anion current-voltage relationship at steady-state is plotted in (B) for ASCT1 and (C) for ASCT2. 


\subsection{Intracellular Substrate Inhibits the Leak Anion Conductance in ASCT1}

Next, we tested the substrate binding properties of the inward-facing state by applying amino acid from the intracellular side of the membrane. This was done using the inside-out patch configuration, allowing the application of intracellular substrate through solution exchange, as previously described for other transporters [35]. From earlier studies with the EAAT SLC1 family members, it is known that intracellular substrate application activates the anion conductance, but with a 20 times lower glutamate apparent affinity (intracellular glutamate apparent affinity is about $100 \mu \mathrm{M}$ compared with $5 \mu \mathrm{M}$ in the outward-facing conformation) [35]. As ionic conditions, we used $\mathrm{SCN}^{-}$in the inside-out recording pipet, together with $140 \mathrm{mM}$ NaMes and $10 \mathrm{mM}$ serine. Thus, the amino acid binding site should be inward-facing before serine was applied to the inside-out patch (Figure 3). As shown in Figure $3 \mathrm{C}$, serine activated the anion conductance in ASCT2, resulting in negative current due to $\mathrm{SCN}^{-}$outflow from the patch pipet [3,36-38]. When increasing the concentration of serine, anion currents increased in a concentration-dependent manner, saturating at high concentrations with an apparent $K_{\mathrm{m}}$ of $70 \pm 18 \mu \mathrm{M}$ (Figure 3D). In contrast, serine application to ASCT1-containing inside-out patches resulted in different behavior (Figure 3A), with substrate blocking the anion conductance, rather than activating it. Similar behavior of blocking the leak anion current was found for intracellular application of a competitive inhibitor (data not shown). From the serine concentration dependence, an apparent $K_{\mathrm{m}}$ value of $77 \pm 34 \mu \mathrm{M}$ was determined Figure 3B, when fit at low substrate concentrations. At concentrations exceeding $500 \mu \mathrm{M}$, a slight decrease in current was observed. The reason for this behavior is not known, although one possibility would be that ASCT1 has an allosteric binding site for the substrate. For ASCT2, these results indicate that substrate binds with slightly higher apparent affinity to the inward-facing conformation, whereas in ASCT1, apparent substrate affinities of the inward and outward-facing states are similar.
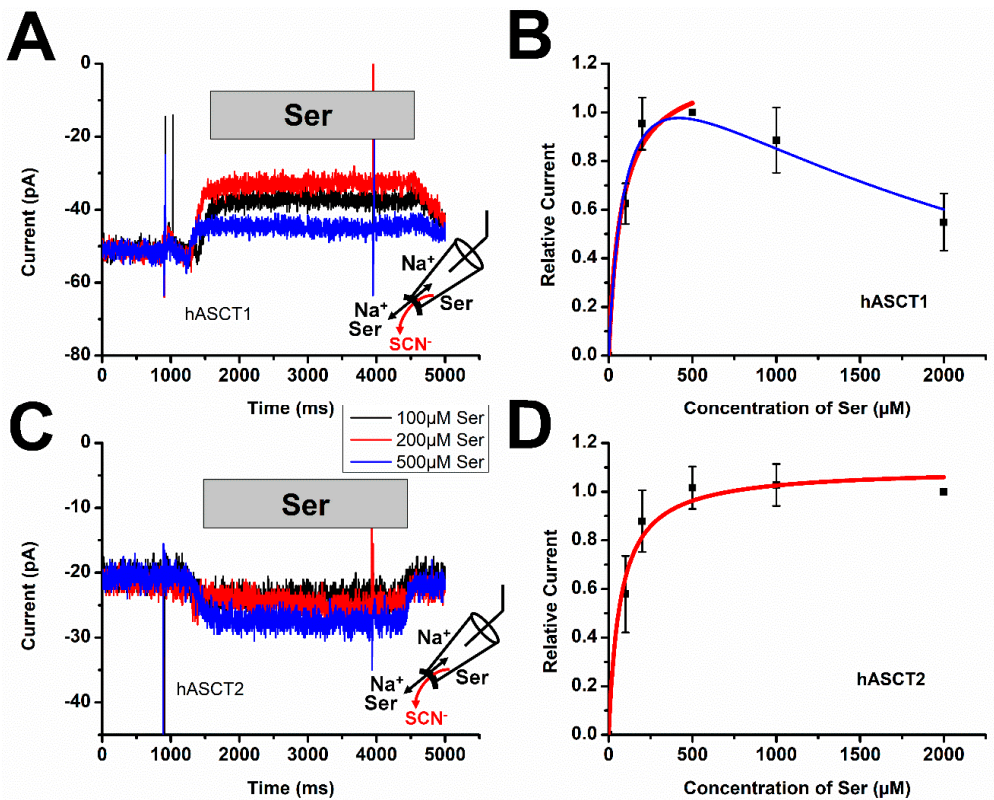

Figure 3. Determination of substrate apparent affinity of the inward-facing state. Representative whole cell current recordings from ASCT1 (A) and 2 (C) transfected cells in the inside-out patch configuration. The extracellular solution (pipet solution) contained $130 \mathrm{mM} \mathrm{NaSCN}$ with $10 \mathrm{mM}$ Ser. The intracellular (bath) solution contained $140 \mathrm{mM}$ NaMes with varying concentrations of serine. (B) Concentration dependence of serine-induced current for determining the apparent affinity for serine of the inward-facing state $\left(K_{\mathrm{m}}=77 \pm 34 \mu \mathrm{M}\right.$ with one site binding fitting equation, or $K_{1}=105 \pm 12 \mu \mathrm{M}, K_{2}=2400 \pm 200 \mu \mathrm{M}$ for two binding site fitting for ASCT1). (D) Determination of apparent affinity of serine of the inward-facing state with a $K_{\mathrm{m}}$ of $70 \pm 18 \mu \mathrm{M}$ for ASCT2. 


\subsection{ASCT Activity Shows Biphasic $\mathrm{Na}^{+}$Concentration Dependence}

Evidence from molecular dynamics simulation and electrophysiology studies [1,2,5,8,39-41], points to the existence of three sodium coordination sites in ASCT1 and 2. By analyzing $\mathrm{Na}^{+}$ dependence of amino acid-induced anion currents, we determined the $\mathrm{Na}^{+}$apparent affinity for both subtypes over a large $\left[\mathrm{Na}^{+}\right]$range, as shown in Figure 4. Total cation concentration was maintained at $140 \mathrm{mM}$ by balancing with $\mathrm{NMG}^{+}$. As observed previously for ASCT2, apparent outward-directed currents were generated by applying amino acid substrate at very low $\mathrm{Na}^{+}$concentration, ranging from 0.1 to $1 \mathrm{mM}$ for both ASCT1 and 2 (Figure $4 \mathrm{~A}, \mathrm{D}$ ), although the magnitude of the outward current was much smaller in ASCT1, presumably due to its lower leak anion conductance (see above). This apparent outward current is caused by a block of the tonic leak anion current (which is inward directed) by the amino acid substrate at low $\left[\mathrm{Na}^{+}\right]$. At higher $\mathrm{Na}^{+}$concentrations, the current direction reversed to generate inward current (substrate-induced anion conductance (Figure 4B,E)). When plotted against the $\mathrm{Na}^{+}$concentration (Figure $4 \mathrm{C}, \mathrm{F}$ ), a bi-phasic current-[ $\left.\mathrm{Na}^{+}\right]$relationship was observed, indicating that more than one $\mathrm{Na}^{+}$binding step is required $[5,8,21]$. With fitting to a two-binding site equation, as shown by the red curves in Figure $4 \mathrm{C}, \mathrm{F}$, the following $K_{\mathrm{m}}$ values for $\mathrm{Na}^{+}$binding were observed: hASCT2, $K_{\mathrm{Na} 1}=0.3 \pm 0.1 \mathrm{mM}$, $K_{\mathrm{Na} 2}=27 \pm 8 \mathrm{mM} ;$ hASCT1, $K_{\mathrm{Na} 1}=0.3 \pm 0.5 \mathrm{mM}, K_{\mathrm{Na} 2}=14 \pm 3 \mathrm{mM}$, indicating slightly weaker $\mathrm{Na}^{+}$apparent affinity for the second cation binding steps in ASCT2 compared to ASCT1.
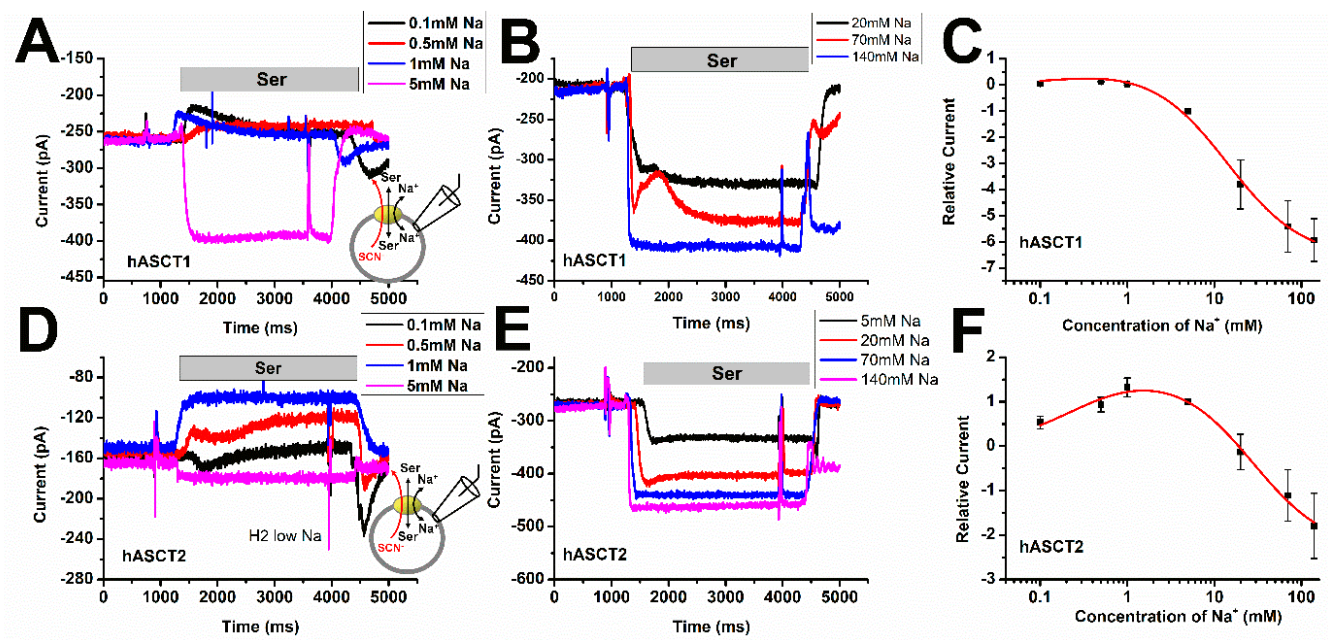

Figure 4. ASCT1 has slightly higher apparent affinity for $\mathrm{Na}^{+}$association to the serine-bound transporter than ASCT2. Whole-cell current recordings were performed to test the apparent affinity for $\mathrm{Na}^{+}$of ASCT transporters. (A,D) Typical serine-induced ASCT1 and 2 anion currents, measured under homo-exchange conditions at varying low $\left[\mathrm{Na}^{+}\right](0-5 \mathrm{mM})$. Serine concentration was saturating (10 mM) extracellularly, with $130 \mathrm{mM} \mathrm{NaSCN}$ and $10 \mathrm{mM}$ Ser inside the pipet. The duration of serine application is indicated by the gray bar. (B,E) Similar experiments as in $(\mathbf{A}, \mathbf{C})$, but in a $\left[\mathrm{Na}^{+}\right]$range from $5 \mathrm{mM}$ to $140 \mathrm{mM}$. (C,F) Serine induced maximum currents plotted as a function of $\left[\mathrm{Na}^{+}\right]$. The $K_{\mathrm{m}}$ for $\mathrm{Na}^{+}$was determined from fitting dose-response curves of a two $\mathrm{Na}^{+}$binding site function. $I=I_{\max 1} *\left[\mathrm{Na}^{+}\right] /\left(K_{\mathrm{Na} 1}+\left[\mathrm{Na}^{+}\right]\right)+I_{\max 2}{ }^{*}\left[\mathrm{Na}^{+}\right] /\left(K_{\mathrm{Na} 2}+\left[\mathrm{Na}^{+}\right]\right)$. For ASCT2, $K_{\mathrm{Na} 1} 0.3 \pm 0.1 \mathrm{mM}$, $K_{\mathrm{Na} 2}$ is $27.9 \pm 8.8 \mathrm{mM}$. For ASCT1, $K_{\mathrm{Na} 1}$ is $0.3 \pm 0.1 \mathrm{mM}, K_{\mathrm{Na} 2}$ is $14.0 \pm 3.4 \mathrm{mM}$. In all experiments, the transmembrane potential was $0 \mathrm{mV}$.

To further test the $\mathrm{Na}^{+}$dependence of ASCT activity, we measured the substrate apparent affinities as a function of the $\mathrm{Na}^{+}$concentration. As shown in Supplementary Materials Figure S1, apparent substrate $K_{\mathrm{m}}$ values decrease with increasing extracellular $\mathrm{Na}^{+}$concentration, as expected for cooperative binding of amino acid substrate and $\mathrm{Na}^{+}$ and as supported by the fits to a sequential $\mathrm{Na}^{+}$-substrate- $\mathrm{Na}^{+}$binding model (solid lines in Supplementary Materials Figure S1). 


\subsection{ASCT1 and 2 Have Comparable Kinetics of Substrate Translocation}

The kinetics of reaction steps associated with ASCT2 substrate translocation were previously studied using rapid alanine application with laser photolysis of caged-alanine $[5,8,34,42]$. In this previous study, transient currents induced by alanine application decayed with a time constant of $3 \mathrm{~ms}$, providing information about the electrogenic steps in the translocation equilibrium [20-22,43-45]. Here, we extended these experiments using a piezo-based rapid solution exchange device, allowing the rapid application, as well as removal of amino acid. These experiments were performed in the absence of permeating anions, to isolate electrogenic steps of the process. The intracellular solution contained $\mathrm{Na}^{+}$and amino acid and, initially, the extracellular solution contained only $\mathrm{Na}^{+}$, conditions under which the amino acid binding site should be facing to the extracellular side of the membrane. Subsequently, serine was applied to the extracellular side of the membrane and later removed. As expected for electroneutral amino acid exchange, we did not observe any steady-state current. However, substantial transient inward current associated with cation binding and/or movement of the charged transport domain was caused by serine application $[8,22]$ in both ASCT1 and 2, as shown in Figure 5A-D. The time constant for current decay was 4-5 ms after serine application. However, the kinetics of this decay could be partially limited by the rate of solution exchange, in particular for removal of the substrate. As expected, the transient currents were absent in the absence of extracellular $\mathrm{Na}^{+}$, suggesting that $\mathrm{Na}^{+}$is a requirement for the electrogenic rearrangement of the ASCT binding sites within the membrane. Upon the removal of serine, transient current was observed in the opposite, outward direction, as expected for the re-equilibration of the translocation equilibrium to the outward-facing conformation, resulting in capacitive-like charge movement (i.e., the charge that moved inward during amino acid application has to move outward upon amino acid removal). Consistently, the outward charge movement upon substrate removal was of the same magnitude as the inward charge movement upon substrate application.
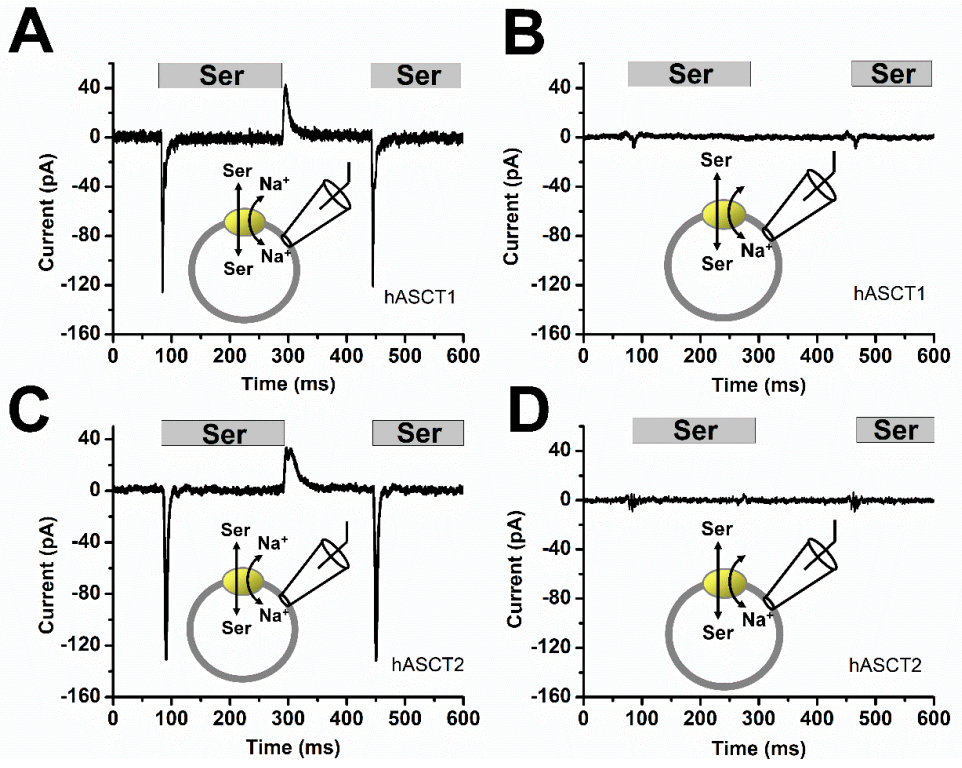

Figure 5. Rapid substrate application and removal induce capacitive-like transient currents in both ASCT1 and 2. (A,C) Typical current recording traces upon rapid solution exchange and removal using a $\theta$ tube. Experiments were performed with $140 \mathrm{mM} \mathrm{Na}^{+}$together with $500 \mu \mathrm{M}$ serine at the extracellular side, the intracellular solution contained $130 \mathrm{mM}$ NaMes and $10 \mathrm{mM}$ serine. Time constants for the decay of the transient current were $\tau_{1}=2.3 \pm 0.1 \mathrm{~ms}$ for serine application and $\tau_{2}=7.8 \pm 0.2 \mathrm{~ms}$ for serine removal (ASCT1) and the charge movements $\mathrm{Q}_{1}=290 \mathrm{fC}$ and $\mathrm{Q}_{2}=340 \mathrm{fC}$, respectively. For ASCT2 the time constants were $\tau_{1}=3.1 \pm 0.1 \mathrm{~ms}$ and $\tau_{2}=11.4 \pm 0.3 \mathrm{~ms}$. (B,D) Similar experiments but in the absence of external $\mathrm{Na}^{+}$. 
Next, we performed a paired-pulse experiment, where the time interval between removal of serine to a second application of serine was varied. This experiment can test how quickly the outward-facing conformation is restored after serine removal, providing information about the amino acid exchange turnover rate of ASCTs. As shown in Figure 6A,D, the peak serine-induced inward current recovered relatively rapidly after serine removal, with complete recovery after 50-100 ms for both transporters. We next performed a fit of the recovery amplitude with an exponential function. The rate constant for recovery was $60 \pm 4 \mathrm{~s}^{-1}$ for ASCT1 (Figure 6B). and $75 \pm 3 \mathrm{~s}^{-1}$ for ASCT2 (Figure 6E), suggesting that the recovery time constant is similar for both ASCT subtypes. Furthermore, we tested time constants at varying substrate concentrations, from $200 \mu \mathrm{M}$ to $1000 \mu \mathrm{M}$, showing that the recovery rate remained unchanged (Figure $6 \mathrm{C}, \mathrm{F}$ ). This is expected because the re-equilibration of the exchange equilibrium should only depend on intracellular substrate concentration after removal of extracellular amino acid.
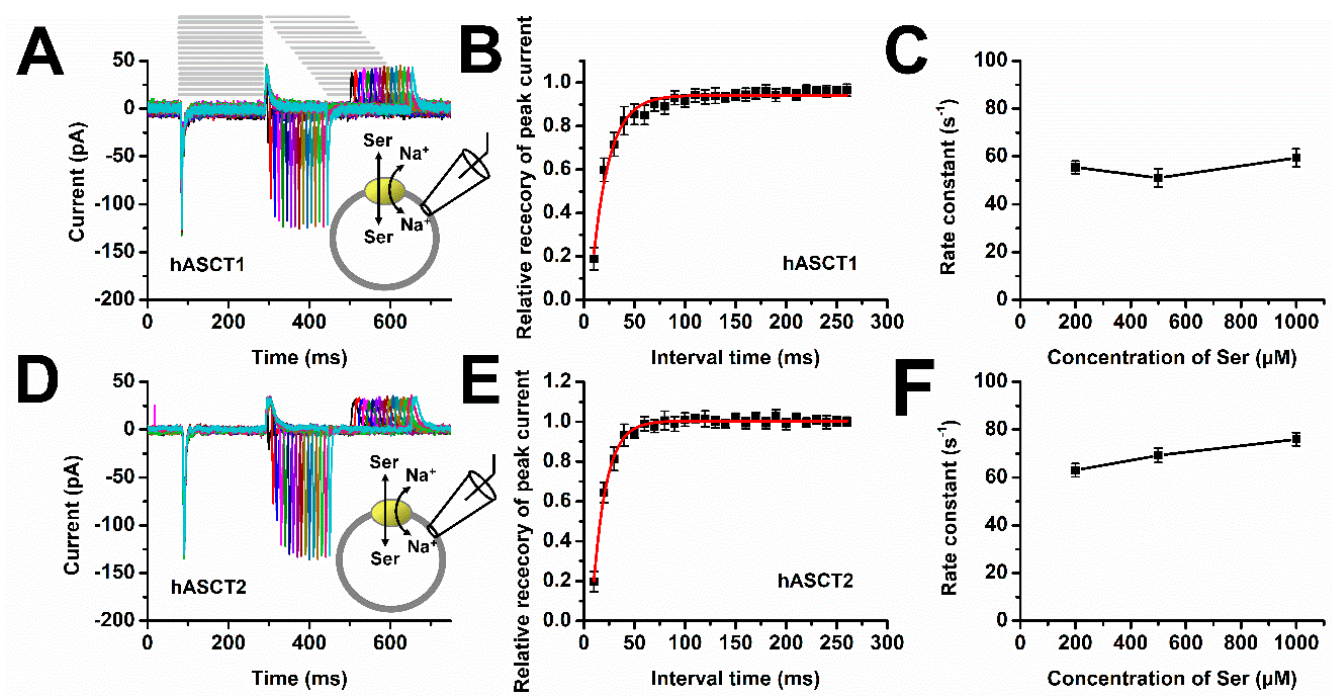

Figure 6. Paired-pulse experiment reveals recovery time constant after substrate removal. Pairedpulse experiments were performed with $140 \mathrm{mM} \mathrm{NaMes}$ and 200,500, or $1000 \mu \mathrm{M}$ serine in the extracellular solution and with $130 \mathrm{mM}$ NaMes and $10 \mathrm{mM}$ serine in the pipet solution. (A,D) Typical set of currents with an increase of recovery time of $10 \mathrm{~ms}$ in each trace (the bars at the top show the solution change protocol) at $500 \mu \mathrm{M}$ serine. Peak amplitudes from (A,B) recovery currents were fitted using a single-exponential function $\left(\mathrm{I}=\mathrm{I}_{0}(1-\exp (-\mathrm{kt}))(\mathbf{B})\right.$, ASCT1 and (E) ASCT2, yielding recovery rate constants of $60 \pm 4 \mathrm{~s}^{-1}$ (B) and $75 \pm 3 \mathrm{~s}^{-1}$ (E). The recovery rate constant was independent of the extracellular serine concentration for both ASCT1 (C) and $2(\mathbf{F})$.

In addition, we tested kinetics associated with the substrate translocation step using different transported amino acids. As shown in Supplementary Materials Figure S2, rate constants associated with recovery from serine and alanine application are in the same order of magnitude, but vary slightly for amino acid and subtype, with rate constants for ASCT2 being larger. Recovery rates after glutamine application are slower and no signal was observed for ASCT1, for which glutamine does not function as a transported substrate (Supplementary Figure S2).

Finally, we determined the voltage dependence of substrate-induced transient currents, at membrane potentials varying from $-100 \mathrm{mV}$ to $+60 \mathrm{mV}$ (Supplementary Materials Figure S3). Peak amplitudes of the transient current signal were plotted as a function of the membrane potential in Supplementary Materials Figure S3A,D. While voltage dependence of the peak current is weak for ASCT2, a stronger voltage dependence was observed for ASCT1, with current increasing about 1.07 fold for every $-20 \mathrm{mV}$ change in membrane potential (Supplementary Materials Figure S3B,E). As expected, the current increased at more negative potential, due to positive charge of the cotransported $\mathrm{Na}^{+}$and/or loaded 
transport domain moving inward within the membrane. Overall, these results are consistent with a previous report [8], which did not find a strong voltage-dependent behavior of amino acid induced transient ASCT2 current.

\section{Discussion}

In this report, we compare the function of the neutral amino acid transporter members of the SLC1 family, ASCT1 and 2, using detailed electrophysiological and kinetic analysis. Despite minor differences in amino acid substrate specificity, with ASCT1 unable to recognize glutamine, the general functional properties are highly conserved between the two transporters. ASCT1 and 2 both catalyze a substrate-induced anion conductance, which is uncoupled with substrate transport. While both transporters also exhibit a leak anion conductance, in the absence of amino acid substrate, this leak conductance is much less pronounced in ASCT1 compared to ASCT2. Second, both transporter subtypes exhibit a biphasic response to $\mathrm{Na}^{+}$, suggesting that the sequential $\mathrm{Na}^{+}$-amino acid- $\mathrm{Na}^{+}$binding mechanism is conserved. However, the apparent affinity of the $\mathrm{Na}^{+}$binding step following substrate binding is somewhat lower in ASCT2 compared to ASCT1. Third, apparent affinities for the amino acid substrate serine binding to the inward-facing binding site are in the sub-100 $\mu \mathrm{M}$ range. However, serine is an inhibitor of the leak anion conductance in ASCT1 when applied to the intracellular side of the membrane, in contrast to ASCT2, where it is an activator. Finally, electrogenic transport steps in the translocation reaction are conserved in ASCT1 and 2 and occur with similar rates, suggesting that the turnover rates of the two transporter subtypes for amino acid exchange are $>65 \mathrm{~s}^{-1}$.

Amino acid and $\mathrm{Na}^{+}$binding were proposed to occur in a sequential fashion, similar to the EAAT members of the family, with at least one $\mathrm{Na}^{+}$binding to the apo-form of the transporter and another $\mathrm{Na}^{+}$binding to the amino acid-bound form. This sequential binding sequence appears to be conserved in both ASCT subtypes. In addition, both transporters show the biphasic $\mathrm{Na}^{+}$dose response relationship, indicating that the $\mathrm{Na}^{+}$ binding step to the empty transporter is of high affinity in both ASCT1 and 2, with an apparent $K_{\mathrm{m}}$ value $<1 \mathrm{mM}$. This suggests that this cation binding step is always saturated under physiological conditions, unless $\left[\mathrm{Na}^{+}\right]$would reach exceedingly low values. In contrast to ASCT2, the block of leak anion current by substrate at low sodium concentrations is not very pronounced, in agreement with the idea that the leak anion conductance is small in ASCT1. If there is a physiological significance for this difference in function is unknown. The $\left[\mathrm{Na}^{+}\right]$binding step following substrate binding is of lower affinity, in the $20 \mathrm{mM}$ range, although this step is also close to being saturated at physiological $\left[\mathrm{Na}^{+}\right]$. This finding is in agreement with the hypothesis that $\mathrm{Na}^{+}$plays a modulatory role in ASCTs, as it is not providing a driving force for amino acid exchange. Overall, these data are in excellent agreement with a recent report on ASCT2 showing a $\mathrm{Na}^{+} /$glutamine stoichiometry of 2:1 and, for the first time, directly demonstrating sodium flux across the membrane during amino acid transport [46].

The binding properties for amino acid from the intracellular side (inward-facing conformation) had not been previously characterized for ASCTs using electrophysiological analysis. However, using radiolabeled substrates uptake assay with sided proteoliposomes, Indiveri and colleagues were able to determine both the internal and external $K_{\mathrm{m}}$ for ASCT2 for several amino acids [32,47,48]. Interestingly, in their assay, serine had a 100-fold higher $K_{\mathrm{m}}$ in the inside-out proteoliposome configuration. In contrast, our data suggest that the internal and external $K_{\mathrm{m}}$ values are in the same 50-100 $\mu \mathrm{M}$ range. The reason for this discrepancy is not known. One possibility would be that Indiveri and colleagues reconstituted purified human ASCT2 in proteoliposomes, whereas our experiments were performed in HEK293 cells, which may contain regulatory factors that are absent in the purified transporter. Second, it is possible that the experimental $K_{\mathrm{m}}$ values differ for activation of the anion conductance and for actual substrate transport. In any case, the potential symmetry/asymmetry of amino acid affinities of the exchanger binding sites has strong implications on our understanding of ASCT function in the physiological 
environment and needs to be investigated more thoroughly. Another interesting result from the inside-out patch experiments is that serine inhibits the leak anion conductance, rather than activating an anion conductance, as is the case in ASCT2.

In a previous report, it was shown that rapid amino acid application to ASCT2 resulted in inwardly-directed transient currents, most likely caused by the movement of charged $\mathrm{Na}^{+}$ into the transport domain, or by structural changes of the charged transport domain due to amino acid translocation, or both [5]. While rate constants for amino acid translocation were determined in this previous work, the turnover rate was only estimated. Here, we extended this previous analysis by using a paired-pulse amino acid application protocol, allowing the determination of the recovery rate of the current upon amino acid removal. This recovery rate is a better indicator of turnover rate than the rate constant of transient current after amino acid application, since the latter only informs on electrogenic partial reactions. The recovery rate was independent of extracellular serine concentration, as expected, but changed when the nature of the amino acid substrate was varied. For serine, a recovery rate constant of $75 \mathrm{~s}^{-1}$ was obtained for ASCT2 and $60 \mathrm{~s}^{-1}$ for ASCT1 (Figure 6), providing a lower limit for the turnover rate, since a slight limitation of these rate constants by solution exchange cannot be fully excluded. In any case, these values provide an estimate of amino acid exchange rates by these SLC1 family subtypes, suggesting that ASCT2 is a marginally faster exchanger than ASCT1. The knowledge of these turnover rates will have substantial implications for our understanding of the impact of ASCTs in physiological, as well as pathophysiological processes related to the metabolic turnover of neutral amino acids, in particular glutamine.

In summary, the work presented here compares the functional and kinetic properties of the neutral amino acid transporters ASCT1 and 2 from the SLC1 family. Both transporters share the high affinity for $\mathrm{Na}^{+}$in the apo-, amino acid-free configuration, suggesting that this $\mathrm{Na}^{+}$binding site (likely the $\mathrm{Na} 3$ site) is always occupied under physiological conditions. In contrast, the $\mathrm{Na}^{+}$binding step following amino acid binding has a much lower apparent affinity, in the 15 to $25 \mathrm{mM}$ range. Both transporters show sub-millimolar apparent affinities for serine in the inward-facing conformation, suggesting a lack of sidedness with respect to substrate affinities. Finally, despite overall electroneutral amino acid exchange, both transporters display fast electrogenic charge movement in response to rapid amino acid application, most likely caused by rearrangements of the transport domain during substrate translocation, or $\mathrm{Na}^{+}$binding. From the recovery of these charge movements after amino acid removal, the turnover rate was estimated in the range of $>65 \mathrm{~s}^{-1}$ for ASCT2 and only slightly slower for ASCT1. Together these data may be useful to understand the involvement of ASCTs in amino acid homeostasis under physiological conditions, as well as in certain cancer cells, in which ASCT2 is upregulated.

Supplementary Materials: The following supporting information can be downloaded at: https: / / www.mdpi.com/article/10.3390/biom12010113/s1, Figure S1: Apparent affinity for substrate is dependent on $\left[\mathrm{Na}^{+}\right]$; Figure S2: Transient current recovery rates depend on the substratetitle; Figure S3: Substrate-induced transient currents show low voltage dependencetitle.

Author Contributions: Conceptualization, J.W., Y.D. and C.G.; methodology, J.W., Y.D.; validation, J.W., Y.D. and C.G.; formal analysis, J.W., Y.D. and C.G.; investigation, J.W., Y.D.; data curation, J.W., Y.D.; writing - original draft preparation, J.W., Y.D. and C.G.; writing-review and editing, J.W., Y.D. and C.G.; visualization, J.W. and Y.D.; supervision, C.G.; project administration, J.W. and C.G.; funding acquisition, C.G. All authors have read and agreed to the published version of the manuscript.

Funding: This study was supported by a grant from the National Institutes of Health (R01 GM108911) to Avner Schlessinger and Christof Grewer and the R15 GM135843-01 awarded to Christof Grewer.

Data Availability Statement: Original data can be found in the supplementary information or will be made available upon request.

Conflicts of Interest: The authors declare no conflict of interest. 


\section{References}

1. Arriza, J.L.; Kavanaugh, M.P.; Fairman, W.A.; Wu, Y.N.; Murdoch, G.H.; North, R.A.; Amara, S.G. Cloning and expression of a human neutral amino acid transporter with structural similarity to the glutamate transporter gene family. J. Biol. Chem. 1993, 268, 15329-15332. [CrossRef]

2. Utsunomiya-Tate, N.; Endou, H.; Kanai, Y. Cloning and functional characterization of a system ASC-like Na+-dependent neutral amino acid transporter. J. Biol. Chem. 1996, 271, 14883-14890. [CrossRef]

3. Zerangue, N.; Kavanaugh, M.P. ASCT-1 is a neutral amino acid exchanger with chloride channel activity. J. Biol. Chem. 1996, 271, 27991-27994. [CrossRef] [PubMed]

4. Broer, A.; Wagner, C.; Lang, F.; Broer, S. Neutral amino acid transporter ASCT2 displays substrate-induced Na+ exchange and a substrate-gated anion conductance. Biochem. J. 2000, 346, 705-710. [CrossRef]

5. Zander, C.B.; Albers, T.; Grewer, C. Voltage-dependent processes in the electroneutral amino acid exchanger ASCT2. J. Gen. Physiol. 2013, 141, 659-672. [CrossRef] [PubMed]

6. Kanai, Y. Substrate binding sites of glutamate transporters and structurally related neutral amino acid transporters. Jpn. J. Physiol. 1997, 47, S55-S56.

7. Foster, A.C.; Farnsworth, J.; Lind, G.E.; Li, Y.X.; Yang, J.Y.; Dang, V.; Penjwini, M.; Viswanath, V.; Staubli, U.; Kavanaugh, M.P. D-Serine Is a Substrate for Neutral Amino Acid Transporters ASCT1/SLC1A4 and ASCT2/SLC1A5, and Is Transported by Both Subtypes in Rat Hippocampal Astrocyte Cultures. PLoS ONE 2016, 11, e0156551. [CrossRef]

8. Zhang, Z.; Papageorgiou, G.; Corrie, J.E.; Grewer, C. Pre-steady-state currents in neutral amino acid transporters induced by photolysis of a new caged alanine derivative. Biochemistry 2007, 46, 3872-3880. [CrossRef]

9. Broer, A.; Brookes, N.; Ganapathy, V.; Dimmer, K.S.; Wagner, C.A.; Lang, F.; Broer, S. The astroglial ASCT2 amino acid transporter as a mediator of glutamine efflux. J. Neurochem. 1999, 73, 2184-2194.

10. Son, J.; Lyssiotis, C.A.; Ying, H.; Wang, X.; Hua, S.; Ligorio, M.; Perera, R.M.; Ferrone, C.R.; Mullarky, E.; Shyh-Chang, N.; et al. Glutamine supports pancreatic cancer growth through a KRAS-regulated metabolic pathway. Nature 2013, 496, 101-105. [CrossRef]

11. Wahi, K.; Holst, J. ASCT2: A potential cancer drug target. Expert. Opin. Targets. 2019, 23, 555-558. [CrossRef]

12. Kanai, Y.; Clemencon, B.; Simonin, A.; Leuenberger, M.; Lochner, M.; Weisstanner, M.; Hediger, M.A. The SLC1 high-affinity glutamate and neutral amino acid transporter family. Mol. Aspects. Med. 2013, 34, 108-120. [CrossRef]

13. Heimer, G.; Marek-Yagel, D.; Eyal, E.; Barel, O.; Oz Levi, D.; Hoffmann, C.; Ruzzo, E.K.; Ganelin-Cohen, E.; Lancet, D.; Pras, E.; et al. SLC1A4 mutations cause a novel disorder of intellectual disability, progressive microcephaly, spasticity and thin corpus callosum. Clin. Genet. 2015, 88, 327-335. [CrossRef]

14. Scalise, M.; Console, L.; Cosco, J.; Pochini, L.; Galluccio, M.; Indiveri, C. ASCT1 and ASCT2: Brother and Sister? SLAS Discov. 2021, 26, 1148-1163. [CrossRef]

15. Bendahan, A.; Armon, A.; Madani, N.; Kavanaugh, M.P.; Kanner, B.I. Arginine 447 plays a pivotal role in substrate interactions in a neuronal glutamate transporter. J. Biol. Chem. 2000, 275, 37436-37442. [CrossRef]

16. Wang, J.; Li, P.; Yu, X.; Grewer, C. Observing spontaneous, accelerated substrate binding in molecular dynamics simulations of glutamate transporters. PLOS ONE 2021, 16, e0250635. [CrossRef]

17. Qiu, B.; Matthies, D.; Fortea, E.; Yu, Z.; Boudker, O. Cryo-EM structures of excitatory amino acid transporter 3 visualize coupled substrate, sodium, and proton binding and transport. Sci. Adv. 2021, 7, eabf5814. [CrossRef] [PubMed]

18. Wang, J.; Zielewicz, L.; Grewer, C. A $\mathrm{K}(+) / \mathrm{Na}(+)$ co-binding state: Simultaneous versus competitive binding of $\mathrm{K}(+)$ and $\mathrm{Na}(+)$ to glutamate transporters. J. Biol. Chem. 2019, 294, 12180-12190. [CrossRef]

19. Wang, J.; Zhang, K.; Goyal, P.; Grewer, C. Mechanism and potential sites of potassium interaction with glutamate transporters. J. Gen. Physiol. 2020, 152, e202012577. [CrossRef] [PubMed]

20. Grewer, C.; Watzke, N.; Wiessner, M.; Rauen, T. Glutamate translocation of the neuronal glutamate transporter EAAC1 occurs within milliseconds. Proc. Natl. Acad. Sci. USA 2000, 97, 9706-9711. [CrossRef] [PubMed]

21. Watzke, N.; Bamberg, E.; Grewer, C. Early intermediates in the transport cycle of the neuronal excitatory amino acid carrier EAAC1. J. Gen. Physiol. 2001, 117, 547-562. [CrossRef] [PubMed]

22. Zhang, Z.; Tao, Z.; Gameiro, A.; Barcelona, S.; Braams, S.; Rauen, T.; Grewer, C. Transport direction determines the kinetics of substrate transport by the glutamate transporter EAAC1. Proc. Natl. Acad. Sci. USA 2007, 104, 18025-18030. [CrossRef] [PubMed]

23. Mim, C.; Balani, P.; Rauen, T.; Grewer, C. The glutamate transporter subtypes EAAT4 and EAATs 1-3 transport glutamate with dramatically different kinetics and voltage dependence but share a common uptake mechanism. J. Gen. Physiol. 2005, 126, 571-589. [CrossRef]

24. Arriza, J.L.; Eliasof, S.; Kavanaugh, M.P.; Amara, S.G. Excitatory amino acid transporter 5, a retinal glutamate transporter coupled to a chloride conductance. Proc. Natl. Acad. Sci. USA 1997, 94, 4155-4160. [CrossRef] [PubMed]

25. Gameiro, A.; Braams, S.; Rauen, T.; Grewer, C. The discovery of slowness: Low-capacity transport and slow anion channel gating by the glutamate transporter EAAT5. Biophys. J. 2011, 100, 2623-2632. [CrossRef]

26. Huang, S.; Ryan, R.M.; Vandenberg, R.J. The role of cation binding in determining substrate selectivity of glutamate transporters. J. Biol. Chem. 2009, 284, 4510-4515. [CrossRef]

27. Borre, L.; Kanner, B.I. Coupled, but not uncoupled, fluxes in a neuronal glutamate transporter can be activated by lithium ions. $J$. Biol. Chem. 2001, 276, 40396-40401. [CrossRef] 
28. Garaeva, A.A.; Oostergetel, G.T.; Gati, C.; Guskov, A.; Paulino, C.; Slotboom, D.J. Cryo-EM structure of the human neutral amino acid transporter ASCT2. Nat. Struct. Mol. Biol. 2018, 25, 515-521. [CrossRef]

29. Garaeva, A.A.; Guskov, A.; Slotboom, D.J.; Paulino, C. A one-gate elevator mechanism for the human neutral amino acid transporter ASCT2. Nat. Commun. 2019, 10, 3427. [CrossRef]

30. Yu, X.; Plotnikova, O.; Bonin, P.D.; Subashi, T.A.; McLellan, T.J.; Dumlao, D.; Che, Y.; Dong, Y.Y.; Carpenter, E.P.; West, G.M.; et al. Cryo-EM structures of the human glutamine transporter SLC1A5 (ASCT2) in the outward-facing conformation. eLife 2019, 8 , e48120. [CrossRef]

31. Garibsingh, R.A.; Ndaru, E.; Garaeva, A.A.; Shi, Y.; Zielewicz, L.; Zakrepine, P.; Bonomi, M.; Slotboom, D.J.; Paulino, C.; Grewer C.; et al. Rational design of ASCT2 inhibitors using an integrated experimental-computational approach. Proc. Natl. Acad. Sci. USA 2021, 118, e2104093118. [CrossRef] [PubMed]

32. Scalise, M.; Pochini, L.; Panni, S.; Pingitore, P.; Hedfalk, K.; Indiveri, C. Transport mechanism and regulatory properties of the human amino acid transporter ASCT2 (SLC1A5). Amino. Acids. 2014, 46, 2463-2475. [CrossRef] [PubMed]

33. Singh, K.; Tanui, R.; Gameiro, A.; Eisenberg, G.; Colas, C.; Schlessinger, A.; Grewer, C. Structure activity relationships of benzylproline-derived inhibitors of the glutamine transporter ASCT2. Bioorg. Med. Chem. Lett. 2017, 27, 398-402. [CrossRef]

34. Shi, Y.; Wang, J.; Ndaru, E.; Grewer, C. Pre-steady-state Kinetic Analysis of Amino Acid Transporter SLC6A14 Reveals Rapid Turnover Rate and Substrate Translocation. Front. Physiol. 2021, 12, 777050. [CrossRef] [PubMed]

35. Watzke, N.; Grewer, C. The anion conductance of the glutamate transporter EAAC1 depends on the direction of glutamate transport. FEBS Lett. 2001, 503, 121-125. [CrossRef]

36. Machtens, J.P.; Kortzak, D.; Lansche, C.; Leinenweber, A.; Kilian, P.; Begemann, B.; Zachariae, U.; Ewers, D.; de Groot, B.L.; Briones, R.; et al. Mechanisms of anion conduction by coupled glutamate transporters. Cell 2015, 160, 542-553. [CrossRef]

37. Wang, J.; Albers, T.; Grewer, C. Energy Landscape of the Substrate Translocation Equilibrium of Plasma-Membrane Glutamate Transporters. J. Phys. Chem. B 2018, 122, 28-39. [CrossRef]

38. Chen, I.; Pant, S.; Wu, Q.; Cater, R.J.; Sobti, M.; Vandenberg, R.J.; Stewart, A.G.; Tajkhorshid, E.; Font, J.; Ryan, R.M. Glutamate transporters have a chloride channel with two hydrophobic gates. Nature 2021, 591, 327-331. [CrossRef]

39. Tao, Z.; Zhang, Z.; Grewer, C. Neutralization of the aspartic acid residue Asp-367, but not Asp-454, inhibits binding of Na+ to the glutamate-free form and cycling of the glutamate transporter EAAC1. J. Biol. Chem. 2006, 281, 10263-10272. [CrossRef]

40. Huang, Z.; Tajkhorshid, E. Identification of the third $\mathrm{Na}+$ site and the sequence of extracellular binding events in the glutamate transporter. Biophys. J. 2010, 99, 1416-1425. [CrossRef]

41. Heinzelmann, G.; Bastug, T.; Kuyucak, S. Free energy simulations of ligand binding to the aspartate transporter Glt(Ph). Biophys. J. 2011, 101, 2380-2388. [CrossRef] [PubMed]

42. Corrie, J.E.; Munasinghe, V.R.; Trentham, D.R.; Barth, A. Studies of decarboxylation in photolysis of alpha-carboxy-2-nitrobenzyl (CNB) caged compounds. Photochem. Photobiol. Sci. 2008, 7, 84-97. [CrossRef]

43. Grewer, C.; Madani Mobarekeh, S.A.; Watzke, N.; Rauen, T.; Schaper, K. Substrate translocation kinetics of excitatory amino acid carrier 1 probed with laser-pulse photolysis of a new photolabile precursor of D-aspartic acid. Biochemistry 2001, 40, 232-240. [CrossRef]

44. Zielewicz, L.; Wang, J.; Ndaru, E.; Grewer, C.T. Transient Kinetics Reveal Mechanism and Voltage Dependence of Inhibitor and Substrate Binding to Glutamate Transporters. ACS Chem. Biol. 2019, 14, 1002-1010. [CrossRef] [PubMed]

45. Wang, J.; Zielewicz, L.; Dong, Y.; Grewer, C. Pre-Steady-State Kinetics and Reverse Transport in Rat Glutamate Transporter EAAC1 with an Immobilized Transport Domain. Neurochem. Res. 2021. [CrossRef] [PubMed]

46. Mazza, T.; Scalise, M.; Pappacoda, G.; Pochini, L.; Indiveri, C. The involvement of sodium in the function of the human amino acid transporter ASCT2. FEBS Lett. 2021, 595, 3030-3041. [CrossRef]

47. Pingitore, P.; Pochini, L.; Scalise, M.; Galluccio, M.; Hedfalk, K.; Indiveri, C. Large scale production of the active human ASCT2 (SLC1A5) transporter in Pichia pastoris-functional and kinetic asymmetry revealed in proteoliposomes. Biochim. Biophys. Acta 2013, 1828, 2238-2246. [CrossRef]

48. Pochini, L.; Scalise, M.; Galluccio, M.; Indiveri, C. Membrane transporters for the special amino acid glutamine: Structure/function relationships and relevance to human health. Front. Chem. 2014, 2, 61. [CrossRef] [PubMed] 